\title{
Reversible cerebral vasoconstriction syndrome: a case series of 7 patients
}

Margarida Lopes ${ }^{1}$, Eduardo Freitas ${ }^{1,2}$, Ana Filipa Santos ${ }^{1}$, João Pinho ${ }^{1}$, Carla Ferreira ${ }^{1}$

${ }^{1}$ Hospital de Braga, Neurology Department, Braga, Portugal

${ }^{2}$ Hospital Santa Luzia- Unidade Local de Saúde do Alto Minho, Neurology Department, Viana do Castelo, Portugal

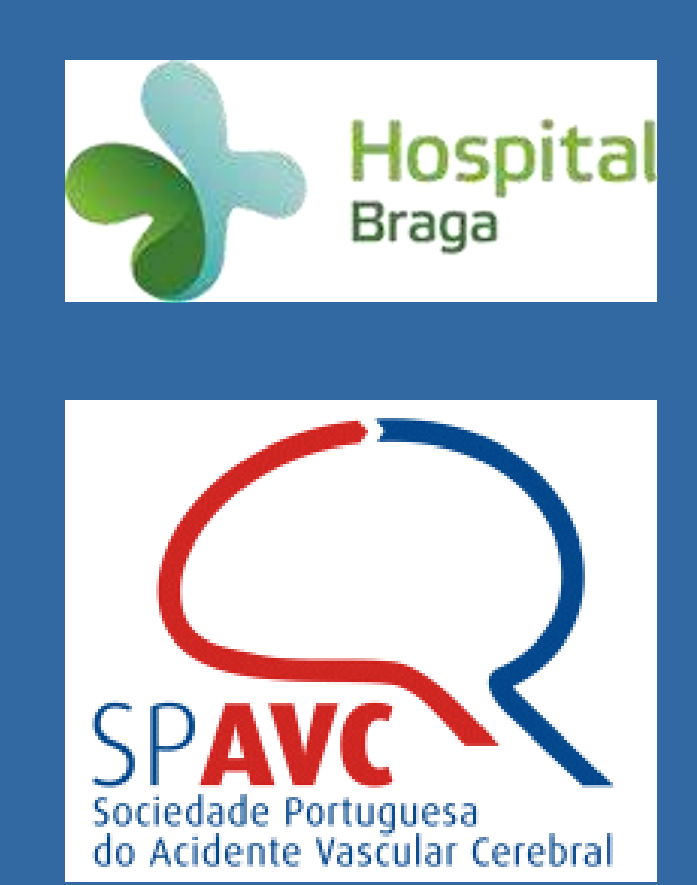

\section{BACKGROUND AND AIMS}

Reversible cerebral vasoconstriction syndrome (RCVS) is characterized by recurrent 'thunderclap' headaches with reversible multifocal narrowing of the cerebral arteries, with or without focal neurological deficits. "Reversible" refers to the dynamic arterial changes seen on angiography, but clinical deficits resulting from infarction or haemorrhage may be persistent. We analysed clinical, neuroimaging and outcome data of patients with angiographically confirmed RCVS.

\section{METHOD}

Retrospective study of patients admitted with the diagnosis of RCVS between August/2015 and September/2017 in our institution

\section{RESULTS}

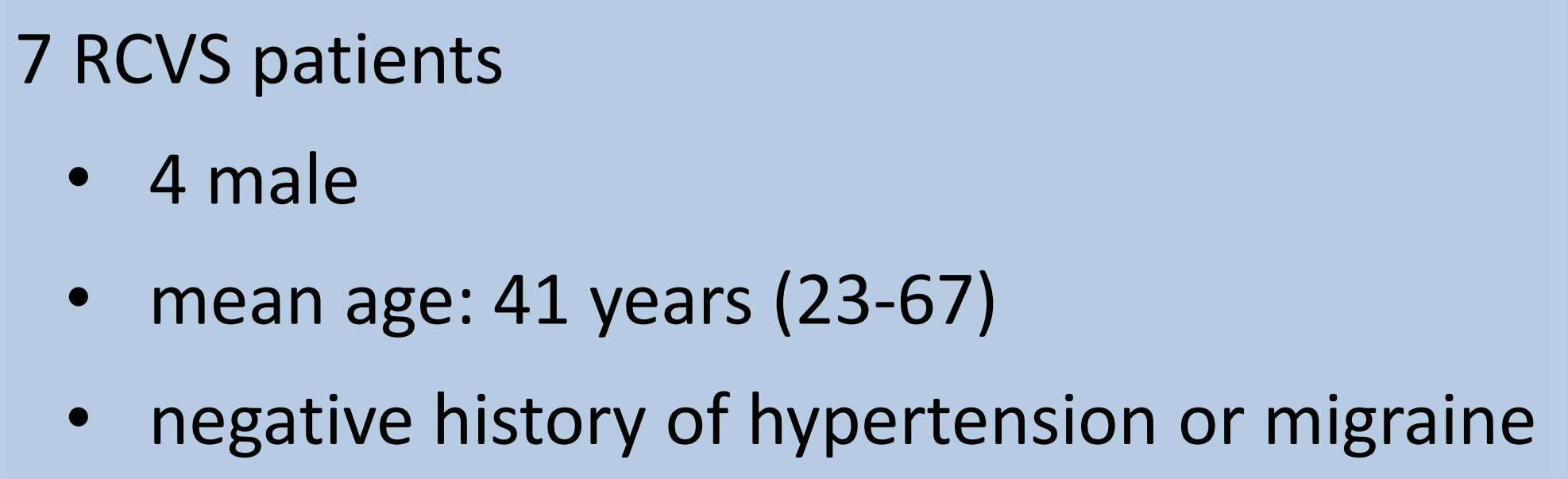

- mean age: 41 years (23-67)

- negative history of hypertension or migraine

Admission blood pressure > 140/90 mmHg: 3 patients mean systolic blood pressure: $187,7 \mathrm{mmHg}$ mean diastolic blood pressure: $96,3 \mathrm{mmHg}$

\begin{tabular}{|ll|}
\hline ASSOCIATED CONDITIONS & $\mathbf{n}$ \\
\hline No identifiable precipitating factor / & $\mathbf{3}$ \\
spontaneous & $\mathbf{2}$ \\
Exposure to vasoactive substances & 1 \\
Cannabinoids & 1 \\
Selective serotonin reuptake inhibitors & $\mathbf{1}$ \\
Puerperium & $\mathbf{1}$ \\
Autonomic dysreflexia (Guillain-Barré syndrome) &
\end{tabular}

\begin{tabular}{|ll|}
\hline DIAGNOSTIC INVESTIGATION & $\mathbf{n}$ \\
\hline Angiographic study & $\mathbf{7}$ \\
CT angiography + MRI angiography & 4 \\
CT angiography + conventional angiography & 1 \\
CT angiography only & 1 \\
MRI angiography only & 1 \\
Brain imaging & $\mathbf{7}$ \\
CT scan + MRI & 5 \\
CT scan only & 1 \\
MRI only & 1 \\
Lumbar puncture & 4 \\
\hline
\end{tabular}

- Mean time [symptoms onset - diagnosis]: 8 days (2-16)

- Mean time [diagnosis - arterial normalization]: 107 days (12-254))
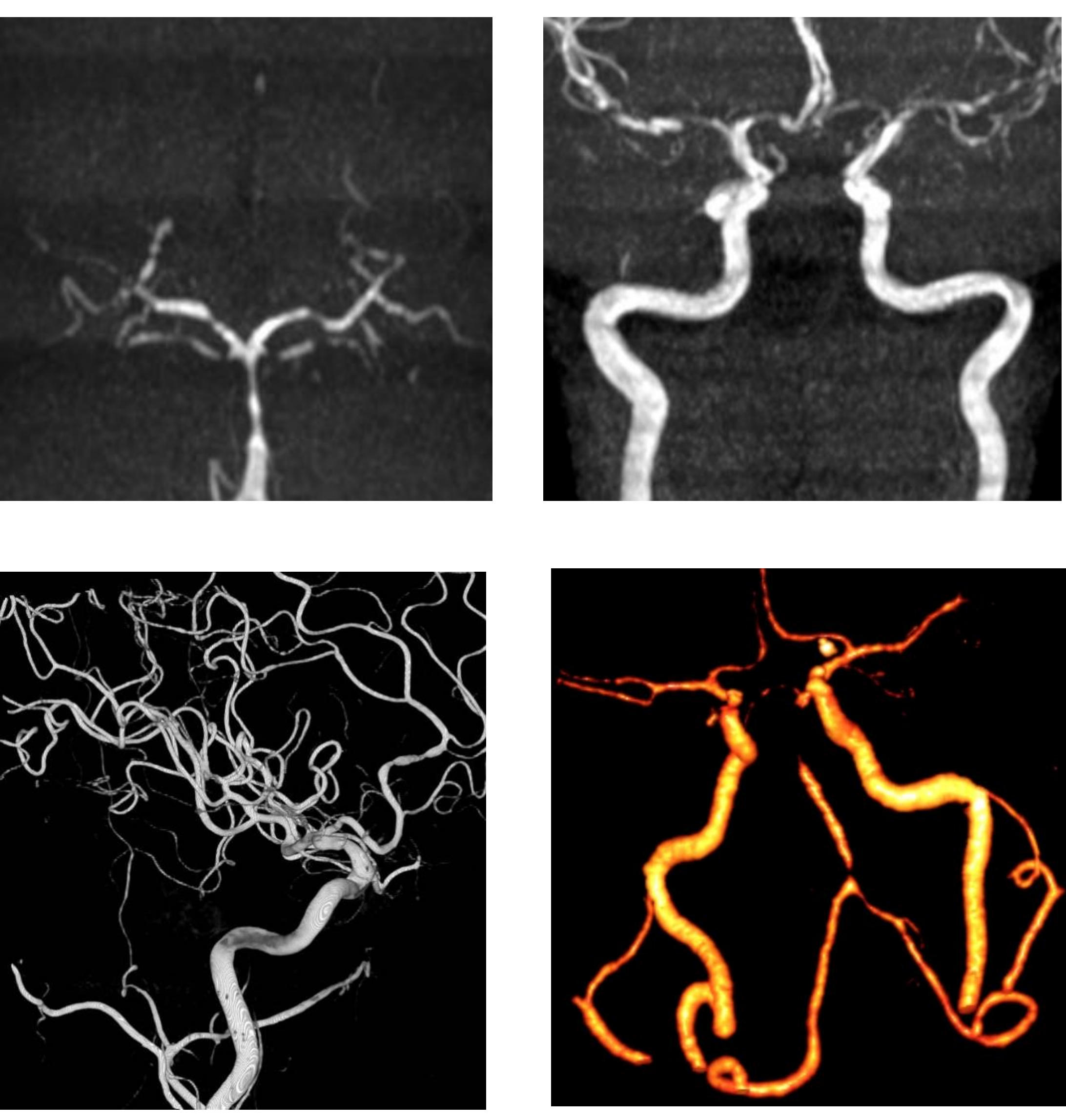

$>$ Three patients with abnormal brain imaging:

- 2 patients with subarachnoid hemorrhage + intra-parenchymal hemorrhage

- 1 patient with cerebral infarction

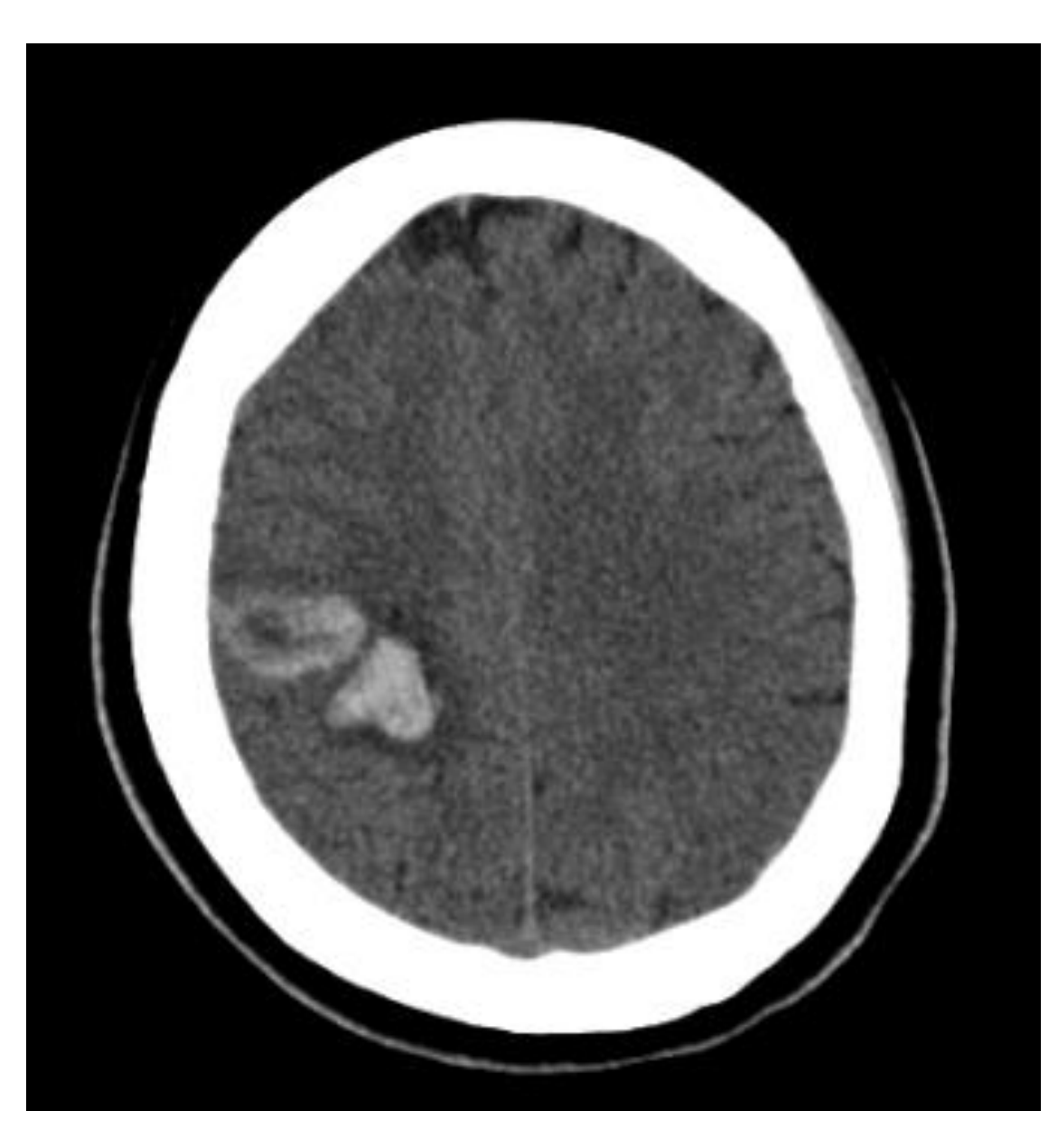

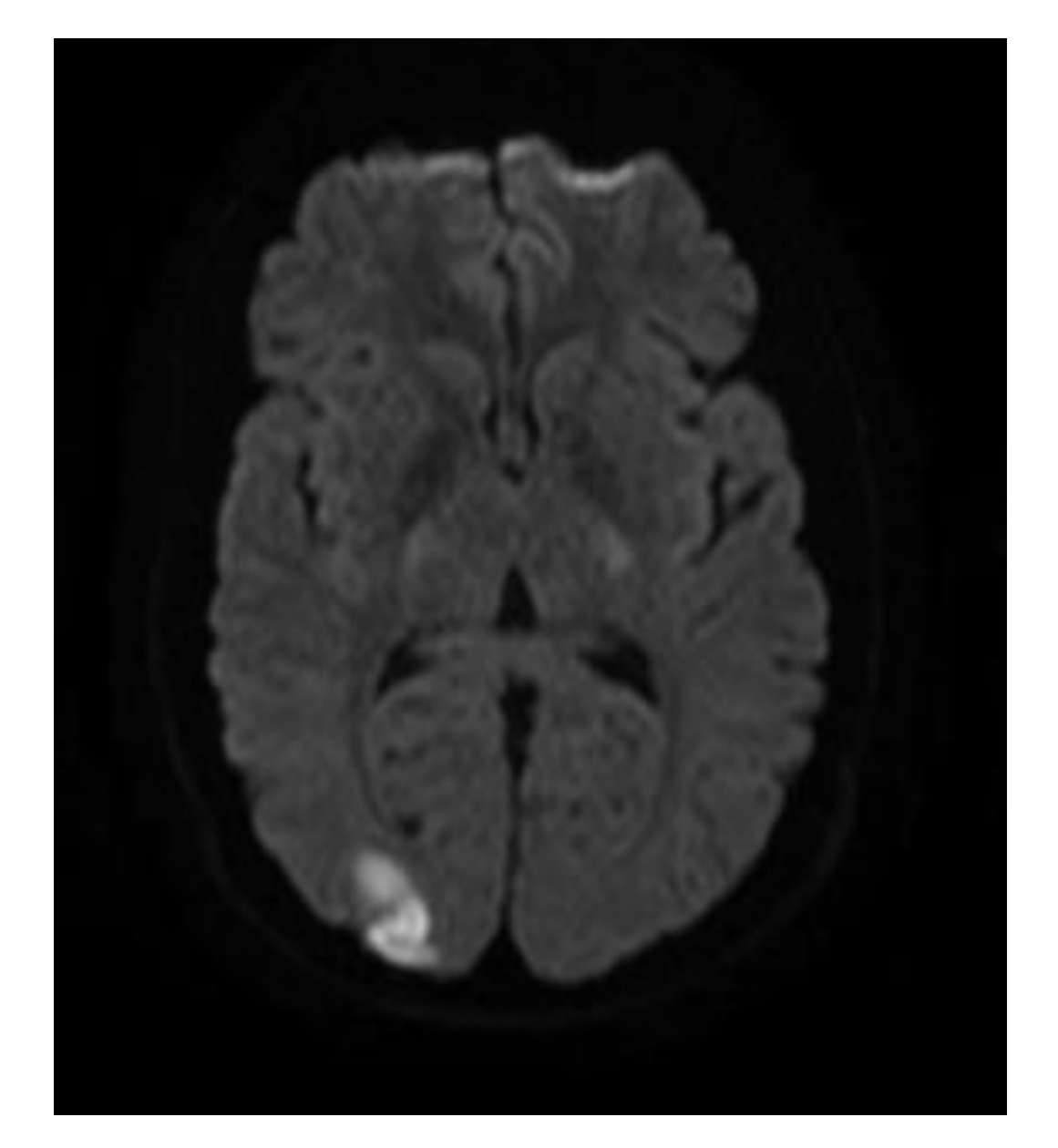

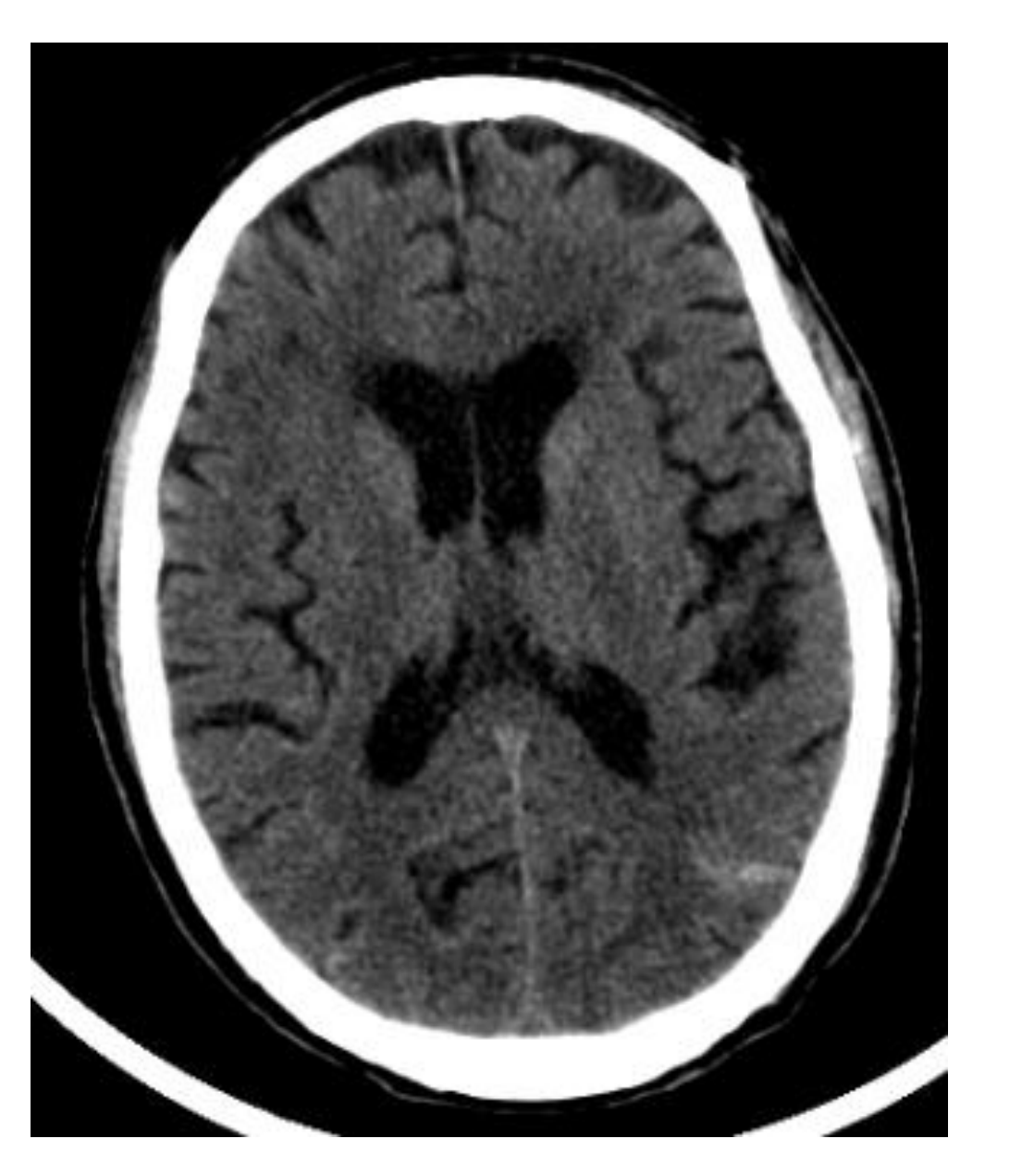

\begin{tabular}{|ll|}
\hline TREATMENT & $\mathbf{n}$ \\
\hline Nimodipine & 5 \\
\hline Acetylsalicylic acid & 3 \\
Corticosteroid / Immunosuppressant & 2 \\
Antiepileptic & 1 \\
Anti-edematous & 1 \\
\hline
\end{tabular}

- No symptomatic recurrence in follow-up No occurrence of death

\section{CONCLUSIONS}

$\checkmark$ RCVS diagnosis is not always obvious, particularly when headaches are the sole clinical presentation or when the first angiographic study is normal.

$\checkmark$ Some cases assumed as primary headaches may be unidentified RCVS, which is why this syndrome should be included in the differential diagnosis of 'thunderclap' headaches, even if the initial investigation is negative. 